\title{
LDA+GTB (Generalized Tight-Binding) method for the electronic structure calculations of strongly correlated electron systems: application for the band structure calculations of p-type cuprates
}

\author{
Maxim M. Korshunov, ${ }^{\text {ab, }}{ }^{*}$ Sergey G. Ovchinnikov ${ }^{\mathrm{b}}$ \\ ${ }^{a}$ L.V. Kirensky Institute of Physics Siberian Branch of RAS, Akademgorodok, 660036 Krasnoyarsk, Russia \\ ${ }^{b}$ Max-Planck-Institut für Physik komplexer Systeme, Nöthnitzer Str. 38, D-01187 Dresden, Germany
}

Elsevier use only: Received date here; revised date here; accepted date here

\begin{abstract}
Mean-field theory of the non-superconducting phase of the High- $T_{c}$ cuprates is formulated within the effective $t-t$ ' $-t$ ''- $J$ model with threesite correlated hoppings. This model with the ab initio calculated parameters results from the LDA+GTB method. The static spin and kinematical correlation functions beyond Hubbard I approximation are calculated self-consistently taking into account hoppings to the first, the second, and the third neighboring sites, as well as the three-site correlated hoppings. The obtained Fermi surface evolves from holepockets at low-doping to large hole-type Fermi surface at higher doping concentrations. Calculated doping dependence of the nodal Fermi velocity, the effective mass and the chemical potential shift are in good agreement with experimental data. C 2001 Elsevier Science. All rights reserved
\end{abstract}

Keywords: Strongly correlated electron systems; superconductivity; p-type cuprates; spin fluctuations; Fermi surface

Recent significant improvements of experimental techniques, especially of the angle-resolved photoemission spectroscopy (ARPES) and the scanning tunneling microscopy (STM), revealed new exciting facts on the lowenergy features of the High- $T_{c}$ copper oxides. First of all, the doping evolution of the Fermi surface has been measured [1]. Then, the dependence of the chemical potential shift $\Delta \mu$ on doping $x$ shows pinning at $x<x_{o p t}$ with $x_{o p t}=0.15$ [2]. The measured nodal Fermi velocity $v_{F}$ is almost doping-independent within experimental error of $20 \%$ [3], together with the effective electron mass $m^{*} / m=3.8 \pm 2$ in $\mathrm{La}_{2-x} \mathrm{Sr}_{x} \mathrm{CuO}_{4}$ and $\mathrm{YBa}_{2} \mathrm{Cu}_{3} \mathrm{O}_{y}$ [4].

Since High- $T_{c}$ cuprates belong to a class of strongly correlated systems, the standard LDA-like schemes and weak-coupling perturbation theories lead to inappropriate results. To overcome this difficulty recently we have formulated an LDA+GTB method [5]. In this method the $a b$ initio LDA calculation is used to construct the Wannier functions and to obtain the single electron and Coulomb parameters of the multiband Hubbard-type model. Within this multiband Hubbard model the electronic structure in the strong correlation regime is calculated by the Generalized Tight-Binding (GTB) method that combines the exact diagonalization of the model Hamiltonian for a small cluster (unit cell) with perturbative treatment of the intercluster hopping and interactions. For undoped $\mathrm{La}_{2} \mathrm{CuO}_{4}$ and $\mathrm{Nd}_{2} \mathrm{CuO}_{4}$ this scheme results in charge transfer insulator with a correct value of the gap and the dispersion of bands in agreement with the experimental ARPES data.

Then the multiband Hamiltonian for the real crystal structure was mapped onto low-energy model. Parameters of this effective model were obtained directly from the $a b$ initio multiband model parameters. The low-energy model appears to be the $t-t^{\prime}-t^{\prime}$ '- $J^{*}$ model $\left(t-t^{\prime}-t^{\prime}\right.$ '- $J$ model with three-cite correlated hoppings) for n-type cuprates and the singlet-triplet $t-t^{\prime}-t^{\prime}{ }^{\prime}-J^{*}$ model for p-type systems. But for

\footnotetext{
"Corresponding author. Tel.: +49-351-8711109; fax: +49-351-8711999; e-mail: maxim@mpipks-dresden.mpg.de.
} 
$x<0.7$ in a phase without long-range order the role of triplet state and singlet-triplet hybridization is negligible [6]. Therefore the triplet could be omitted, and in the present paper we will describe low-energy excitations in the single-layer p-type cuprates within $t-t^{\prime}-t^{\prime} \cdot-J^{*}$ model with $\mathrm{LDA}+\mathrm{GTB}$ calculated parameters (in $\mathrm{eV}$ ): $t=0.93$, $t^{\prime}=-0.12, t^{\prime \prime}=0.15, J=0.295, J^{\prime}=0.003, J^{\prime \prime}=0.007$.

To go beyond the Hubbard I approximation we will calculate electronic Green function using equation of motion method and retain [7] static spin correlation functions $C_{f g}=2\left\langle S_{f}^{z} S_{g}^{z}\right\rangle=\left\langle X_{f}^{\uparrow \downarrow} X_{g}^{\downarrow \uparrow}\right\rangle$ and kinematical correlation functions $K_{f g}=\left\langle X_{f}^{\uparrow 0} X_{g}^{0 \downarrow}\right\rangle$.
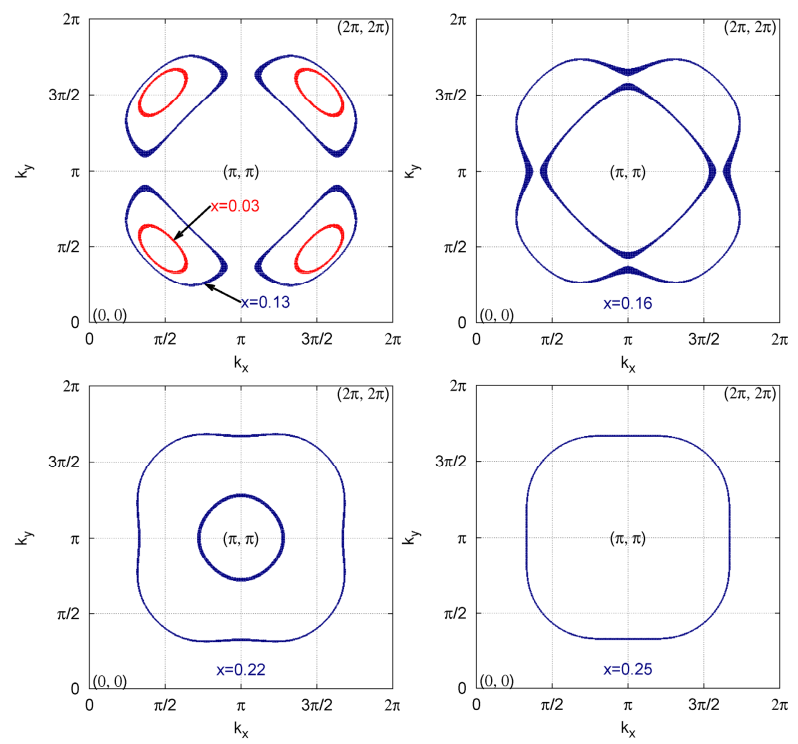

Fig. 1. Fermi surface evolution with doping (hole concentration) $\mathrm{x}$.

The importance of the three-cite correlated hoppings in the normal and superconducting phases has been demonstrated in Refs. [7,8]. To take them into account we use method [9] to self-consistently calculate all $C_{n}$ and $K_{n}$ correlation functions. In the latter method the spinliquid phase is considered and static spin correlation functions calculated using bosonic Green functions similar to $[10]$.

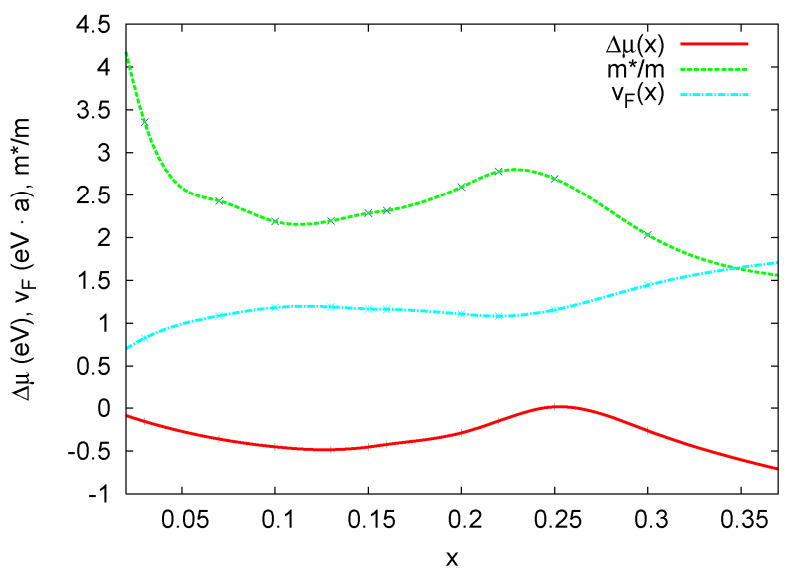

Fig. 2. Doping dependent evolution of the chemical potential shift, nodal Fermi velocity, and effective mass.
Calculated evolution of the Fermi surface (FS) is presented in Fig. 1. At low dopings FS has the form of the hole pockets centered around $( \pm \pi / 2, \pm \pi / 2)$. At higher dopings these pockets merge together and become one large hole-type FS.

Also, we have calculated $v_{F}, m^{*} / m$ and $\Delta \mu$ vs. doping $x$ (see Fig. 2). Nodal Fermi velocity and chemical potential reveals only slight doping dependence in good agreement with the experiments [2,3]. Effective mass $m$ * increase with decreasing $x$ reveals tendency to the localization in the vicinity of the metal-insulator transition. But this increase is not very large and overall $m *$ doping dependence agrees very well with experimentally observed one [4].

To summarize, we have investigated the dopingdependent evolution of the low-energy quantities for p-type High- $T_{c}$ cuprates in the regime of strong electron correlations within sequentially derived effective model with $a b$ initio parameters. Although our approach is a mean-field theory, the calculations within t- $\mathrm{t}$ '-J model including finite quasiparticle lifetime [11] shows that the results of the mean-field-like approximation is qualitatively correct. Quantitatively, at low doping the imaginary part of the self-energy leads to the hiding of the FS portions above the antiferromagnetic Brillouin zone $((\pi, 0)-(0, \pi)$ line $)$. This results in Fermi arc rather than hole pockets at $x<x_{\text {opt }}$ (see Fig. 1).

Authors would like to thank D.M. Dzebisashvili, V.V. Val'kov and N.M. Plakida for very helpful discussions. This work was supported by INTAS YS grant 05-1094891, Siberian Branch of RAS (Lavrent'yev Contest for Young Scientists), RFBR grant 06-02-16100, Program of Physical Branch of RAS "Strongly correlated electron systems", and Joint Integration Program of Siberian and Ural Branches of RAS N.74.

\section{References}

[1] K.M. Shen, F. Ronning, D. H. Lu et al., Science 307 (2005) 901.

[2] N. Harima, J. Matsuno, A. Fujimori et al., Phys. Rev. B 64 (2001) 220507(R).

[3] T. Yoshida, A. Lanzara, P. V. Bogdanov et al., Nature 423 (2003) 398.

[4] W.J. Padilla, Y.S. Lee, M. Dumm et al., Phys. Rev. B 72 (2005) 060511(R).

[5] M.M. Korshunov, V.A. Gavrichkov, S.G. Ovchinnikov et al., Phys. Rev. B 72 (2005) 165104.

[6] M.M. Korshunov, S.G. Ovchinnikov, A.V. Sherman, Phys. Met. Metallogr. 100 (2005) S75.

[7] M.M. Korshunov, S.G. Ovchinnikov, A.V. Sherman, JETP Lett. 80 (2004) 39.

[8] V.V. Val'kov, T.A. Val'kova, D.M. Dzebisashvili et al., JETP Lett. 75 (2002) 378.

[9] V.V. Val'kov, D.M. Dzebisashvili, JETP 100 (2005) 608.

[10] H. Shimahara, S. Takada, J. Phys. Soc. Jpn. 61 (1992) 989.

[11] N.M. Plakida, V.S. Oudovenko, to appear in this volume of Physica C. 